\section{Correlating nerve fibre layer defects spatially with functional loss}

\author{
Abstract \\ The ultimate aim of clinical glaucoma research \\ is to maintain visual function in the patient. \\ Visual field (VF) loss can be determined more \\ effectively by considering the related \\ structural damage (ie retinal nerve fibre layer \\ (RNFL) defect, optic disc notching, and \\ splinter haemorrhages). A stack of \\ individually digitized RNFL traces, which are \\ superimposed by appropriate rotation and \\ zoom procedures, allows for characterizing \\ so-called trajectories defining the average \\ course of retinal nerve fibres. \\ Individual test point density in perimetry can \\ be increased in regions of interest, that is in \\ areas, or at the edges of, already detected VF \\ defects (VFD) using SCotoma-Oriented \\ Perimetry (SCOPE) or can be tailored (locally \\ condensed) in an evidence-based manner by \\ considering the local morphological damage \\ using fundus-oriented perimetry (FOP). \\ Focusing on areas most likely to be damaged \\ makes conventional perimetry more efficient \\ by locally enhancing spatial resolution. This \\ technique requires a mathematical model that \\ provides age-related normative values of \\ differential luminance sensitivity for any \\ location of the VF. Automated static perimetry \\ with enhancement of spatial resolution in \\ regions of interest has indeed a higher \\ sensitivity than standard automated perimetry \\ using a conventional rectangular $\left(6 \times 6^{\circ}\right)$ grid. \\ The new method can also be used for follow- \\ up purposes: both scotoma depth and area, as \\ well as their changes with time, need to be \\ considered. The clinician has to be aware of \\ the fact that any kind of perimetry is a rather \\ exhausting and artificial examination \\ procedure. It is rather inadequate for \\ predicting per se patients' quality of life and \\ daily functionality as it does not consider \\ compensation for VFD, for example by gaze \\ and head movements.
}

U Schiefer ${ }^{1}$, J Paetzold ${ }^{1}$, E Krapp ${ }^{1}$, J Nevalainen ${ }^{1,2}$ and $\mathrm{D}$ Besch ${ }^{1}$

Eye (2007) 21, S25-S28; doi:10.1038/sj.eye.6702884

Keywords: visual field; perimetry; grid;

glaucoma; follow-up; RNFL, retinal nerve fibre layer

\section{Introduction}

The gold standard for detecting functional damage in glaucoma is currently threshold-estimating automated static grid perimetry. Perimetry involves a trade-off between resolution in space and depth, on the one hand, and examination time, on the other. The number of stimulus locations examined per visual field (VF) examination is usually 50-80, within $24-27^{\circ}$ eccentricity from fixation. The stimuli are usually based on a square grid with an inter-stimulus separation of $6^{\circ}$. Such a stimulus grid enables the detection of a focal defect of approximately $7^{\circ}$ in diameter (ie a scotoma the size of the blind spot) with a probability of $95 \%{ }^{1}$

\section{Rationale for using condensed grids}

Glaucomatous progression is thought to occur in the vicinity of already affected neurons, ${ }^{2-4}$ suggesting that a local progression of scotoma depth and/or size may be the first sign of change. This means that perimetric tests should concentrate, or condense, stimuli at those locations with the highest index of suspicion of glaucomatous damage or progression. By increasing spatial resolution, the detection of a smaller area of field loss is possible. Using individually condensed test grids focussed on morphologically suspicious areas thus increases the sensitivity for VF defect (VFD) detection compared with conventional $\left(6 \times 6^{\circ}\right)$ rectangular stimulus arrangements. ${ }^{5}$ Algorithms can be applied to the construction
${ }^{1}$ Centre for Ophthalmology, University of Tübingen, Tübingen, Germany

${ }^{2}$ University Eye Hospital Oulu, Oulu, Finland

Correspondence: Professor U Schiefer, Centre for Ophthalmology, Schleichstr. 12-16, D-72076 Tübingen, Germany Tel: + 4970712984786 E-mail: ulrich.schiefer@ uni-tuebingen.de 
of a local condensation of the test point, according to the suspected underlying basic pathology - notching of the optic disc, for example.

\section{Retinal nerve fibre layer and fundus-oriented perimetry}

It is problematic to extrapolate from standard automated static grid VF testing, not least because conventional rectangular $6 \times 6^{\circ}$ perimetry tends to cover no more than $1.5 \%$ of the entire $30^{\circ} \mathrm{VF}$ area. Another problem is caused by short and long-term intra-subject variability, which hinders the identification of progressive VF loss in serial VF analysis. To reduce this problem, fundus-oriented perimetry (FOP) has been developed, in which the patient's fundus image is downloaded or digitized onto a control monitor and mirrored, if necessary, with the help of special software (Figure 1).

Assuming central fixation, the foveola of the fundus image is aligned to the centre of the perimetric field using a cross hair. In a second step, the patient's retinal damage (blind spot), which has been determined by kinetic

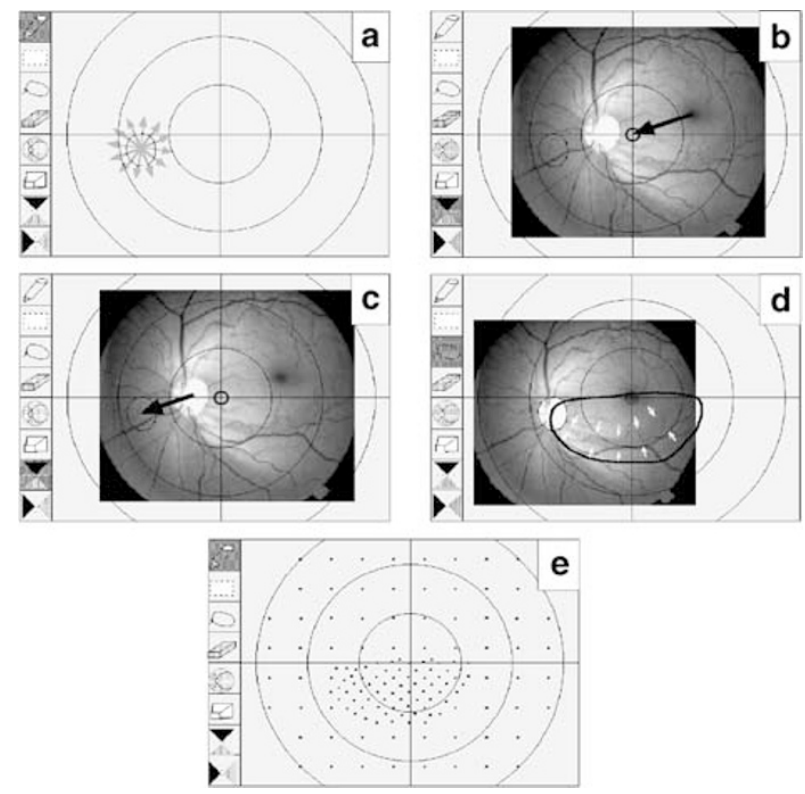

Figure 1 Basic principle of FOP. (a) Assessment of blind spot via manual kinetic perimetry. (b) Loading of individual fundus image, which is then transposed along the horizontal axis and shifted for superimposition of foveola and centre of VF (translational alignment). (c) Superimposition of optic disc and blind spot (rotation and zoom procedure). (d) Selection of individual ROI, including visible RNFL defect (white arrows). (e) Resulting individual perimetric grid with circumscribed stimulus condensation within the inferior hemifield (ROI) and conventional, equidistant, $6 \times 6^{\circ}$ spacing elsewhere. In case of SCOPE local condensation of test points is based on previous field examinations of the patient. (Reproduced from Schiefer et $a l^{6}$ with permission of Arch Ophthalmol, 2003;121:458, Copyright 2003, American Medical Association. All Rights reserved.) perimetry, is interactively superimposed onto the optic disc of the fundus image by automatic activation of rotation and zoom routines, and transposition along the horizontal axis. Accurate superimposition involves registration of the foveola and centre of the VF. The individual region of interest (ROI) can then be selected, including visible retinal nerve fibre layer (RNFL) defects. The result is an individual perimetric grid with circumscribed stimulus condensation within the ROI and conventional, equidistant $6 \times 6^{\circ}$ spacing elsewhere (Figure 1). A stack of individually digitized RNFL traces, which are superimposed by appropriate rotation and zoom procedures, allows the characterization of so-called trajectories defining the average course of retinal nerve fibres. ${ }^{7}$ FOP cannot be applied in case of diffuse structural loss as there is no morphological clue for a test point condensation.

If FOP is not available, individual test point density can be increased in areas, or at the edges of, already detected functional loss (ie VFD) using SCotomaOriented PErimetry (SCOPE). Central fixation is an essential prerequisite for both methods. A deficient or unstable central fixation will result in suboptimal spatiofunctional interrelation and reduced reproducibility.

\section{Mathematical modelling of the hill of vision}

For a clinically successful application of FOP, the age-related normative function of differential luminance sensitivities (DLS) for any point of the VF (including stimulus concentration within regions of interest) needs to be known. ${ }^{8}$ This requires an adequate smooth mathematical model characterizing the entire central $\left(30^{\circ}\right)$ region of the hill of vision (Figure 2$){ }^{9}$

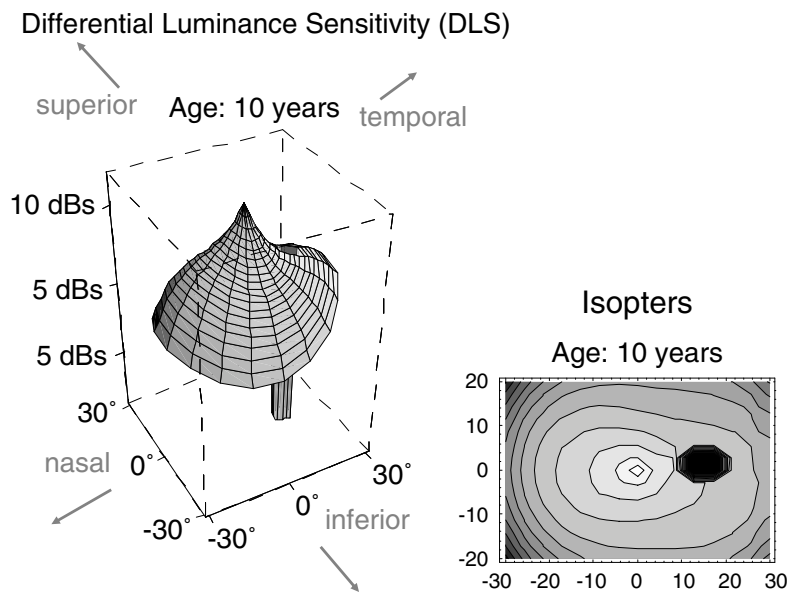

Figure 2 Age-related (10 years of age) hill of vision, obtained by smooth mathematical modelling. 
A time-lapse series, applying this model on the hills of vision over age shows no relevant change up to the fourth decade. At around the age of 40 , the hill of vision will shift, not only its level by moving downwards, but also its configuration similar to incompletely closing an umbrella. ${ }^{10}$

\section{Clinical results of perimetry with spatially enhanced stimulus density}

\section{Detection of glaucomatous VFD}

Application of the method to a series of 66 patients has shown how it can help to detect VFDs, with a higher detection rate than conventional automated perimetry $(\mathrm{CAP})$, using a $\left(6 \times 6^{\circ}\right)$ rectangular grid. ${ }^{6}$ For 15 patients, CAP showed normal findings, but FOP revealed early glaucomatous VFDs. Only one patient showed VFDs with CAP, and normal results with FOP. Scotoma detection rates significantly differed between the two methods ( $P<0.001$, sign test), and repeatability between the two methods comparable.

Langerhorst et $a l^{11}$ demonstrated that higher test point density within the central $10^{\circ} \mathrm{VF}$ enhanced scotoma detection. Westcott et al ${ }^{12-14}$ showed a similar effect by adding test locations within the region of the nasal step. In contrast to FOP, both methods did not adapt stimulus arrangements according to the individual morphological findings, but used default grids thereby eventually wasting time with additional test points in obviously normal regions.

Basically, FOP can be applied in all cases of circumscribed (morphological) loss, for example in pits of the disc, segmental optic atrophy, circumscribed chorioretinal lesions, branch occlusion of retinal arteries. In cases of circumscribed scotomas without visible structural changes, the application of SCOPE may be a useful option.

After superimposing the results of perimetric results with enhanced spatial resolution in a consecutive series of 89 eyes of 75 glaucoma patients, the local spatial frequency distribution of defective field localizations $(P<0.05)$ confirmed a higher frequency of VFDs in the superior, compared with the inferior, hemifield. This preference also occurred in the immediate vicinity of the VF centre, that is glaucomatous optic neuropathy results much more frequently in fixation threat within the superior part than the inferior part (Nevalainen et al, private communication).

Enhanced spatial resolution in ROI is achieved by adding stimulus locations to the original grid. This results in an increase of test duration. As a consequence, the stimulus condensation has to be restricted to circumscribed ROI. In addition, fast thresholding algorithms, which are independent of the stimulus arrangement, are most welcome to keep examination time at a reasonable range. Developments of strategies in this concern are underway. ${ }^{15}$

Thus, FOP has been validated for detecting the existence of VFD, but it is also clinically important to measure disease progression, in terms of VFD depth and size.

\section{Follow-up of glaucomatous VFD}

Careful evaluation of local DLS over time in perimetry, with spatially enhanced stimulus density, reveals that several modes of progression coexist in the VF of the same patient. Progression may become manifest as continuous deterioration of DLS over time, which can be most adequately described by pointwise linear regression in one location. Abrupt deterioration of DLS (ie the event) may occur in another circumscribed area, whereas a third location may indicate the risk of progression by an increased DLS variability (ie neuronal "loose contact"). Increasing the test point density in regions of interest (ie areas of potential deterioration) also reduces the disturbing impact of local variability by also taking into account neighbouring locations.

An alternative approach was shown by the work of Gardiner et $a l^{16}$ in terms of spatial filtering techniques, which exploit this feature to reduce measurement noise.

By applying locally high-spatial resolution perimetry to a series of 69 glaucoma eyes over a time series of at least 2 years (with a minimum of four sessions), approximately two-thirds (43/69) remained unchanged, only $1 \%(1 / 69)$ showed an isolated change in size, $4 \%$ (3/69) demonstrated an isolated change in depth and $32 \%(22 / 69)$ showed a combined change in depth and size. ${ }^{17}$ As a consequence, CAP does not seem to perform optimally in characterizing and quantifying glaucomatous progression: CAP is predominantly used for detection of glaucomatous scotoma progression in depth, whereas a subtle progression in size usually remains undetected because of its comparatively coarse $\left(6 \times 6^{\circ}\right)$ stimulus arrangement.

\section{Conclusion}

Structural data can be assessed in an almost examiner-independent way. However, functional data are irreplaceable with regard to any kind of expert opinion, ability testing and assessment of visual performance. Therefore, imaging and functional diagnostics are not mutually exclusive, but complementary ophthalmological tools. Morphological/ morphometric data can be used to focus functional diagnostic methods to ROI individually and vice versa.

Enhancement of spatial resolution by increasing test point density in ROI has been proven to be an efficient, evidence-based tool in regard to detection and follow-up 
of glaucomatous VF loss. Nevertheless, perimetry remains a rather artificial method. Further effort is needed to predict/forecast the patient's quality of life and visual performance: in this regard, additional items such as compensation of VFDs via gaze and head movements have to be taken into account.

\section{References}

1 Fankhauser F, Bebie H. Threshold fluctuations, interpolations and spatial resolution in perimetry. Docum Ophthal Proc Ser 1979; 19: 295-309.

2 Schwartz M, Lazarov-Spiegler O, Moalem G, Yoles E. Dialog between traumatized optic nerve axons and immune cells: Implications for survival and regrowth. Invest Ophthalmol Vis Sci 1998; 39: 876

3 Yoles E, Schwartz M. Degeneration of spared axons following partial white matter lesion: implications for optic nerve neuropathies. Exp Neurol 1998; 153: 1-7.

4 Schwartz M, Yoles E. Self-destructive and self-protective processes in the damaged optic nerve: implications for glaucoma. Invest Ophthalmol Vis Sci 2000; 41: 349-351.

5 Schiefer U, Malsam A, Flad M, Stumpp F, Dietrich TJ, Paetzold J et al. Evaluation of glaucomatous visual field loss with locally condensed grids using fundus-oriented perimetry (FOP). Eur J Ophthalmol 2001; 11: 57-62.

6 Schiefer U, Flad M, Stumpp F, Malsam A, Paetzold J, Vonthein $\mathrm{R}$ et al. Increased detection rate of glaucomatous visual field damage with locally condensed grids: a comparison between fundus-oriented perimetry and conventional visual field examination. Arch Ophthalmol 2003; 121: 458-465.

7 Schiefer U, Zangwill LM, Sample PA, Budde W, Jonas JB, Lagrèze $\mathrm{W}$ et al. Evaluation of the human retinal nerve fibre bundle (RNFB) course by fitting trajectories to aligned digitised fundus images. Invest Ophthalmol Vis Sci 2005; 46, E-Abstract 615 .

8 Hermann A, Paetzold J, Vonthein R, Krapp E, Rauscher S, Schiefer U. Age-dependent normative values of differential luminance sensitivity in automated static perimetry - a formula converting threshold data obtained with TCC
(Tuebingen Computer Campimeter) and Octopus 101. Meeting of the International Perimetric Society (IPS) 2004.

9 Paetzold J, Rauscher S, Vonthein R, Hermann A, Sadowski B, Nowomiejska KE et al. Age-dependent reference values of semi-automated kinetic perimetry (SKP) with consideration of individual reaction times using the Octopus 101 instrument. Meeting of the International Perimetric Society (IPS) 2004.

10 Schwabe R, Vonthein R, Ata N, Paetzold J, Dietrich TJ, Schiefer U. Modeling the hill of vision. In: Wall M, Mills RP (eds). Perimetry Update 2000/2001. Kugler Publications: The Hague/The Netherlands, 2001, pp 71-79.

11 Langerhorst CT, Carenini LL, Bakker D, De Bie-Raakman MAC. Measurements for description of very early glaucomatous field defects. In: Wall M, Heijl A (eds). Perimetry Update 1996/1997. Kugler: Amsterdam/New York, 1997, pp 67-73.

12 Westcott MC, Garway-Heath DF, Fitzke FW, Kamal D, Hitchings RA. Use of high spatial resolution perimetry to identify scotomata not apparent with conventional perimetry in the nasal field of glaucomatous subjects. $\mathrm{Br} \mathrm{J}$ Ophthalmol 2002; 86: 761-766.

13 Westcott MC, Garway-Heath DF, Fitzke FW, Hitchings RH. Is conventional perimetry sufficient for the evaluation of the nasal step in glaucoma? High spatial resolution perimetry can identify scotomas not apparent on conventional Humphrey testing. Invest Ophthalmol Vis Sci 1999; 40: 581.

14 Westcott MC, McNaught AI, Crabb DP, Fitzke FW, Hitchings RA. High spatial resolution automated perimetry in glaucoma. Br J Ophthalmol 1997; 81: 452-459.

15 Schiefer U, Pascual J, Sample PA, Edmunds B, Weleber R, Johnson C et al. Comparison of the new 'German Adaptive Threshold Estimation' (GATE) strategy with conventional static threshold estimating perimetry and with the SITA procedure. Invest Ophthalmol Vis Sci 2007; ARVO: 2007.

16 Gardiner SK, Crabb DP, Fitzke FW, Hitchings RA. Reducing noise in suspected glaucomatous visual fields by using a new spatial filter. Vis Res 2004; 44: 839-848.

17 Paetzold J, Sample PA, Selig B, Krapp E, Vonthein R, Schiefer U. Differentiation between glaucomatous scotoma progression in depth and in size by using scotoma-oriented perimetry (SCOPE) with locally condensed stimulus arrangements. Invest Ophthalmol Vis Sci 2005; 46, E-Abstract 636. 